\title{
Highly variable chloroplast genome from two endangered Papaveraceae lithophytes Corydalis tomentella and C. saxicola
}

\author{
Fengming Ren ${ }^{1}$, Liqiang Wang ${ }^{2}$, Ying $\mathrm{Li}^{1}$, wei zhuo ${ }^{3}$, Zhichao Xu ${ }^{1}$, Haojie Guo ${ }^{4}$, Yan Liu $^{5}$, \\ Ranran Gao $^{1}$, and Jingyuan Song ${ }^{1}$ \\ ${ }^{1}$ Chinese Academy of Medical Sciences \& Peking Union Medical College Institute of \\ Medicinal Plant Development \\ ${ }^{2}$ Heze University \\ ${ }^{3}$ Chongqing Institute of Medicinal Plant Cultivation \\ ${ }^{4}$ Engineering Research Center of Chinese Medicine Resource, Ministry of Education \\ ${ }^{5}$ Chongqing Institute of Medicinal Plant Cultivation,
}

June 30, 2020

\begin{abstract}
The increasingly wide application of chloroplast(cp) genome super-barcode in taxonomy and the recent breakthrough in $\mathrm{cp}$ genetic engineering make the development of new cp gene resources urgent and significant. Corydalis is recognized as the most genotypes complicated and taxonomically challenging plant taxa in Papaveraceae. However, no complete cp genome for this genus has been reported to date. In this study, we sequenced four complete cp genomes of two endangered lithophytes Corydalis saxicola and C. tomentellav in Corydalis, conducted a comparative genomics study on them, and a highly variable cp genome structure was found. The cp genomes have a large genome size of 189,029 to 190,247 bp, possessing a quadripartite structure and with two highly expanded inverted repeat (IR) regions (length: 41,955 to 42,350 bp). Comparison between the cp genomes of C. tomentella, C. saxicola and Papaveraceae species, five NADH dehydrogenase-like genes (ndhF, ndhD, ndhL, ndhG, ndhE) with psaC, rpl32, ccsA and trnL-UAG normally located in the SSC region have migrated to IRs, resulting in IR expansion and gene duplication. An up to $9 \mathrm{~kb}$ inversion involving five genes (rpl23, ycf2, ycf15, trnI-CAU and trnL-CAA) was found within IR regions. The accD gene was found to be absent and the ycf1 gene has shifted from the IR/SSC border to the SSC region as a single copy. Phylogenetic analysis based on the sequences of common CDS showed that the genus Corydalis is quite distantly related to the other genera of Papaveraceae, it provided a new clue for recent advocacy to establish a separate Fumariaceae family. Our results revealed one special cp genome structure in Papaveraceae, provided a useful resources for classification of the genus Corydalis, and will be valuable for understanding Papaveraceae evolutionary relationships.
\end{abstract}

\section{Hosted file}

Highly variable chloroplast genome from two endangered Papaveraceae lithophytes Corydalis saxicola and available at https://authorea.com/users/338419/articles/464421-highly-variable-chloroplastgenome-from-two-endangered-papaveraceae-lithophytes-corydalis-tomentella-and-c-saxicola 

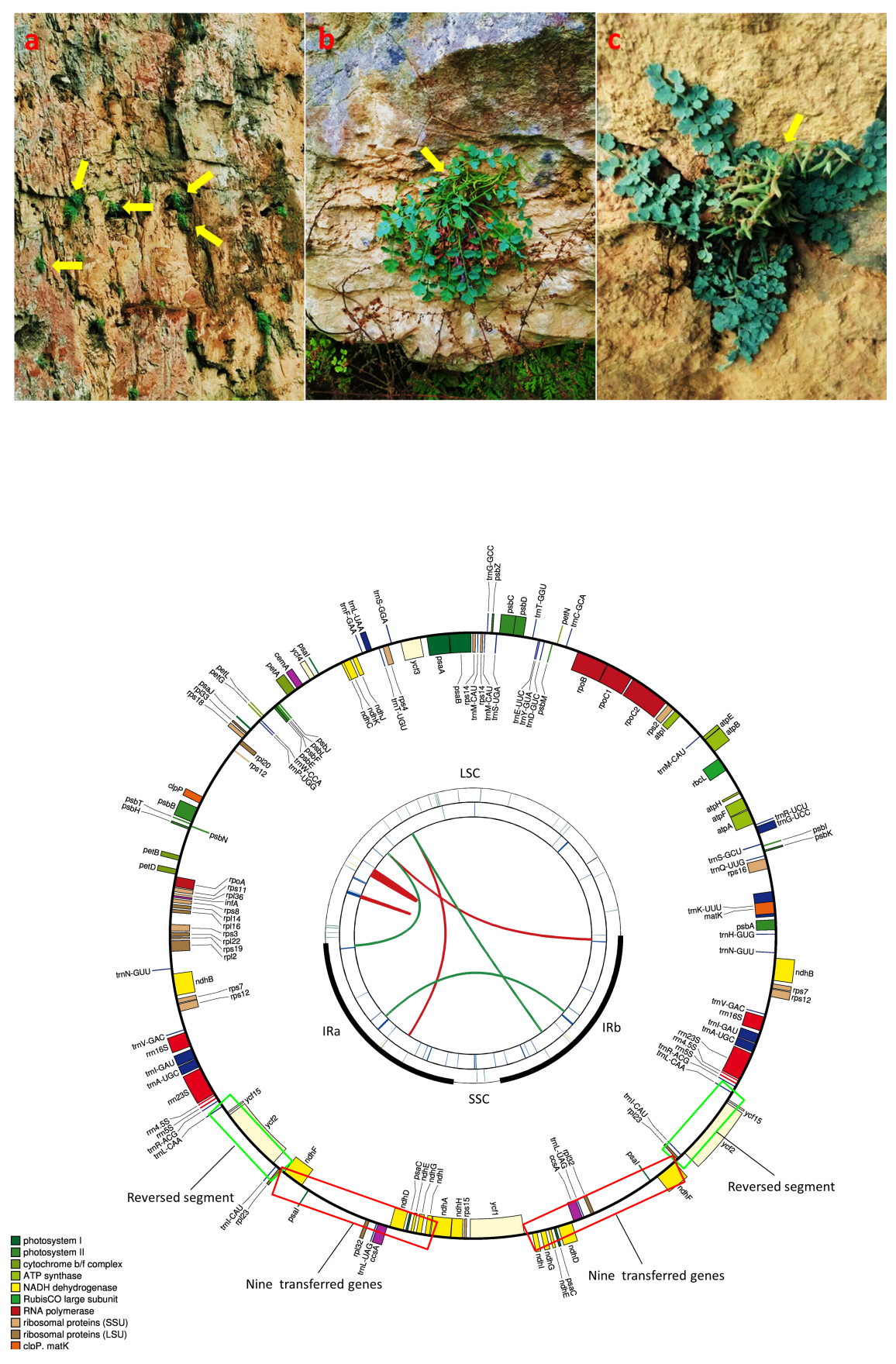

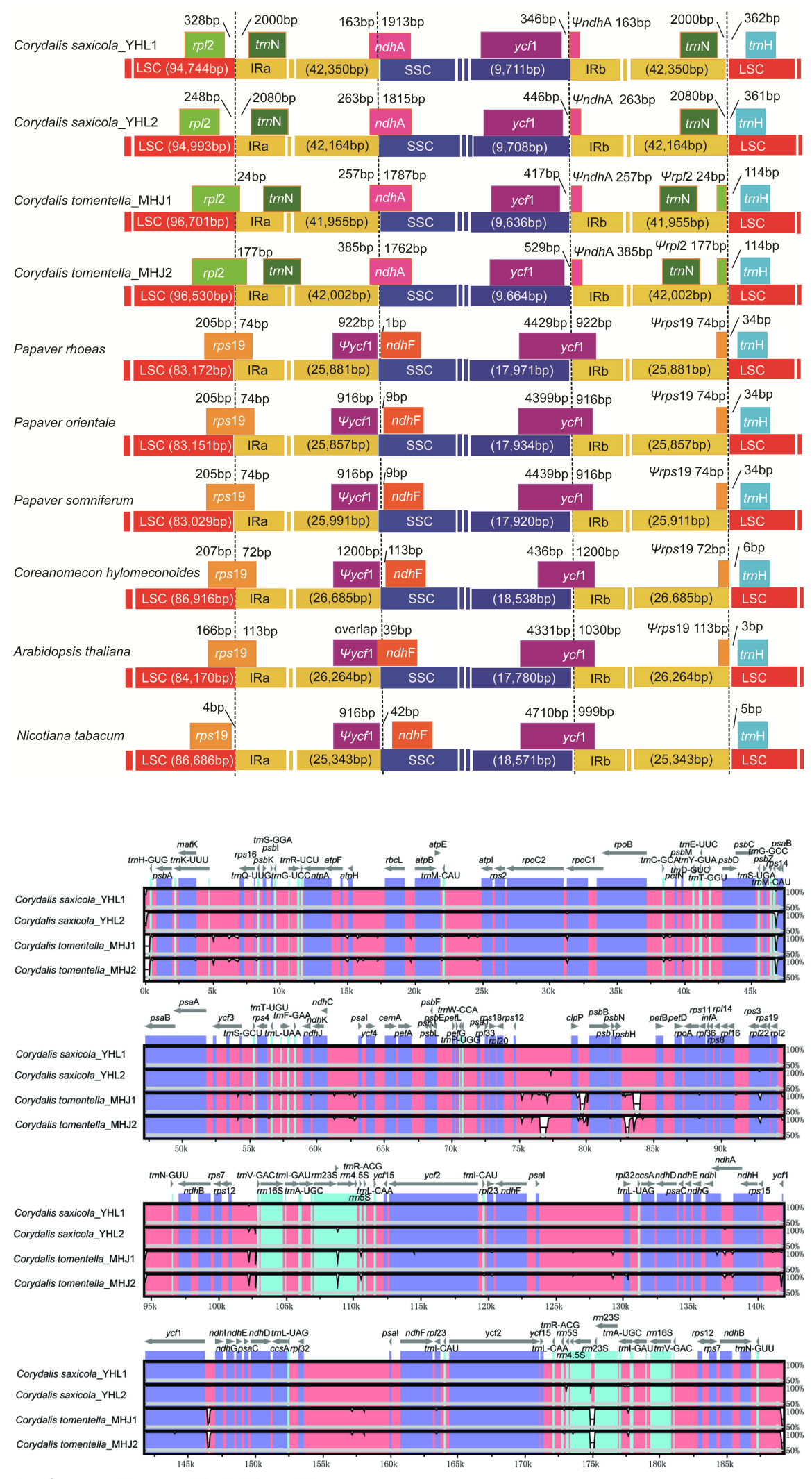

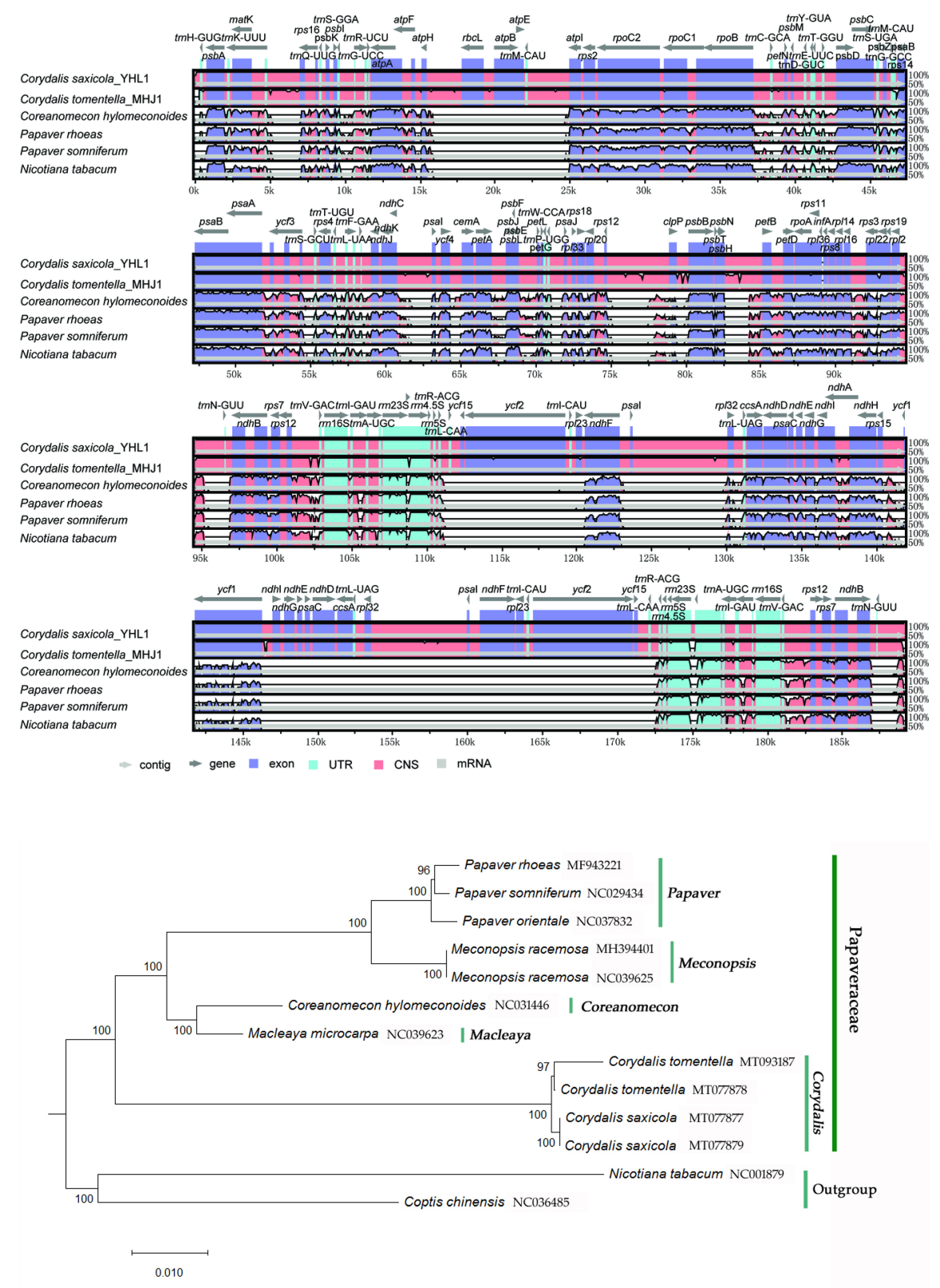